\title{
Evaluation and Comparison of Anti Cholesterol And Antioxidant Potential of Allium Sativum, Zingiber Officinale, Allium Parvum and it's Polyherbal Formulation
}

\author{
B.Sadhvi', Gayathri.R ${ }^{2}$ and Vishnu priya.V ${ }^{3}$ \\ ${ }^{1}$ Graduate student, Saveetha Dental College and Hospitals, Saveetha Institute of \\ Medical and Technical Sciences,Saveetha University, Chennai 77, India \\ ${ }^{2}$ Assistant Professor, Department of Biochemistry, Saveetha dental college and \\ Hospitals, Saveetha Institute of Medical and Technical Sciences,Saveetha University, Chennai 77, India \\ ${ }^{3}$ Professor, Department of Biochemistry, Saveetha Dental College and Hospitals, \\ Saveetha Institute of Medical and Technical Sciences,Saveetha University, Chennai 77, India
}

\begin{abstract}
Cholesterol is an essential sterol found in our body.However, with high cholesterol levels, fatty deposits can develop in the blood vessels and cause heart disorders. Hypercholesterolemia along with Non elimination of free radicals and its accumulation in the body, on the other hand causes serious disorders such as atherosclerosis, cancer, inflammatory joint disease, asthma, diabetes etc. Including some of the naturally available anti cholesterol and antioxidant agents would prevent such disorders in the long run, and this study aims in throwing limelight on three such natural ingredientsginger, garlic and small onions. The aim of this study is to evaluate and compare the anticholesterol and antioxidant potential of Allium sativum, Zingiber officinale, Allium parvum and it's polyherbal formulation. Pure extracts of ginger, garlic and small onions were prepared followed by its antioxidant potential analysis using DPPH Free radical scavenging activity, Nitric oxide radical scavenging activity and In vitro anti cholesterol activity. The results thus obtained were statistically compared with each other and also with the combination of the three extracts. Statistical analysis shows that the allium sativum (garlic) has the individual anti cholesterol and antioxidant activity a little more when compared to the other two, while Zingiber officinale (ginger) shows more antioxidant activity when compared to the other two extracts. Allium parvum (Small onion), has almost equal anti cholesterol and antioxidant activity. The combination of the extracts in the ratio 1:2:1 (garlic:ginger:small onion) shows almost equal anti cholesterol and antioxidant potential when compared to the standard. The above results show that including ginger, garlic and small onion extracts in regular diets can boost health to a great extent and prevent early onset of cardiac related disorders, which are prevalent in the present generation
\end{abstract}

KEY WORDS: ALLIUM SATIVUM, ZINGIBER OFFICINALE, ALLIUM PARVUM, ANTI CHOLESTEROL, ANTIOXIDANT.

\section{ARTICLE INFORMATION}

${ }^{*}$ Corresponding Author: gayathri.sdc@saveetha.com

Received 15th June 2020 Accepted after revision 7th August 2020

Print ISSN: 0974-6455 Online ISSN: 2321-4007 CODEN: BBRCBA

Thomson Reuters ISI Web of Science Clarivate Analytics USA and Crossref Indexed Journal

\section{Clarivate
Analytics}




\section{INTRODUCTION}

Since a protracted time has passed, humans have acknowledged the health advantages of food. (Ferguson and Everett, 1982). However, with the development of chemical drugs and pills, they have forgotten that the food they eat are directly linked to their health (Afzal et al., 2001). Food that include herbal products acts as a remedy for so many prevailing disorders and diseases.Firstly, choosing the right diet and the right food to eat can help improve health and body functions (Prance, Duke and Ayensu, 1985). Secondly, using food for health benefits has been a norm and that scientists are looking forward to creating foods that can fight diseases (Qureshi et al., 1989). Not surprisingly, there are some similarities and differences in the views on the health benefits of foods between two different generations. Moreover, food can also be used as medicine to treat diseases like chemical pills as well(Dugasani et al., 2010).

Cholesterol is an essential sterol found in our body. However, with high cholesterol levels, fatty deposits can develop in the blood vessels and cause heart disorders (Gull et al., 2012) Hypercholesterolemia along with Non elimination of free radicals and its accumulation in the body, on the other hand causes serious disorders such as atherosclerosis, cancer, inflammatory joint disease, asthma, diabetes etc.(Lanzotti et al., 2012) (Leuschner and Ielsch, 2003).

Garlic (Allium sativum) is used as a spice and medicinal herb. Most recent research on garlic has used garlic in the form of tablets, flesh, raw, boiled, cooked and dried (Santas, Almajano and Carbó, 2010). As an herbal remedy commercially available garlic pills and oils are used to lower serum LDL levels and also to decrease hypertension.(Lu et al., 2011) Garlic exhibits a wide range of properties including immunomodulatory hepatoprotective, antimutagenic and anticarcinogenic effects (Li et al., 2017). Garlic and garlic extracts are believed to possess beneficial effects for the prevention of cardiovascular diseases (Micová et al., 2018).Garlic modulates lipid metabolism. Several studies have also shown that garlic contains active hypocholesterolemic and hypoglycemic components, known as diallyl disulfide and dipropyl disulfide(Perry et al., 2009) . It has also been reported that garlic supplements in human subjects lead to the increased resistance of low density lipoprotein to oxidation and may be one of the powerful mechanisms accounting for the antioxidative and anti-atherosclerotic properties of garlic (Piscitelli et al., 2002). With regard to the antioxidative activity of garlic, results have demonstrated that components of aged garlic extract inhibit the in vivo oxidation of LDL by chelating $\mathrm{Cu}+2$, scavenging superoxide ions, thus inhibiting the oxidation of protein and lipid moiety of human LDL-Cholesterol (Gaafar, 2012).

Ginger (Zingiber officinale), a popular spice and vegetable in worldwide, is used as a traditional medicine in China and East Asia countries to treat rheumatism, toothaches, asthma, constipation, and diabetes (Ogodo and Ekeleme, 2013). Many studies on ginger are focused on fresh ginger and their bioactive components. In several studies, ginger and its active compounds have been shown to exert strong antioxidant activities in vitro and in vivo (Singh et al., 2009). Though fresh and dried ginger are used in food and medicine, the antioxidant properties in both differ enormously.Small Onions are strong in flavor and high in polyphenolic compounds called flavonoids. Flavonoids may have anti-inflammatory abilities, antioxidants, anticancer abilities, antiproliferative abilities, or the ability to stop cell growth.

Studies also suggest that small onions may help improve cholesterol levels. In one study, flavonoids in small onions reduced the low-density lipoprotein (LDL), or "bad" cholesterol in obese people at risk of cardiovascular disease. The researchers attributed this to the specific flavonoid quercetin, an antioxidant found in onions and other fruits and vegetables. High-density lipoprotein (HDL), or "good" cholesterol, levels were not affected (Prakash, Singh and Upadhyay, 2007). Another study looked at the effect of small onion extract on cholesterol in rats. The researchers noted a significant decrease in cholesterol levels, though triglyceride levels remained unchanged. Some of the rats were given small onion extract and zinc sulfate, while others were only given small onion extract or zinc sulfate.

Better results were seen among the rats that were given a combination of small onion extract and zinc sulfate (Van Damme et al., 1993). Small onions (allium parvum) may also benefit cholesterol levels. In a previous study done, male hamsters were fed with a diet rich in highcholesterol. Some of the rats' diet was supplemented with small onion powder. The rats that received the small onion powder experienced lower LDL cholesterol levels and maintained high HDL cholesterol levels (Ye, Dai and $\mathrm{Hu}, 2013)$. The rationale of this study is that no study has been conducted so far in which the properties of all the three elements- Ginger (Zingiber officinale), Garlic (Allium sativum L.) and small onions (allium parvum) were reinforced together as a polyherbal formulation and studied for their anticholesterol and antioxidant potential. Hence, this study aims to Evaluate and compare the anticholesterol and antioxidant potential of allium sativum, zingiber officinale, allium parvum and it's polyherbal formulation.

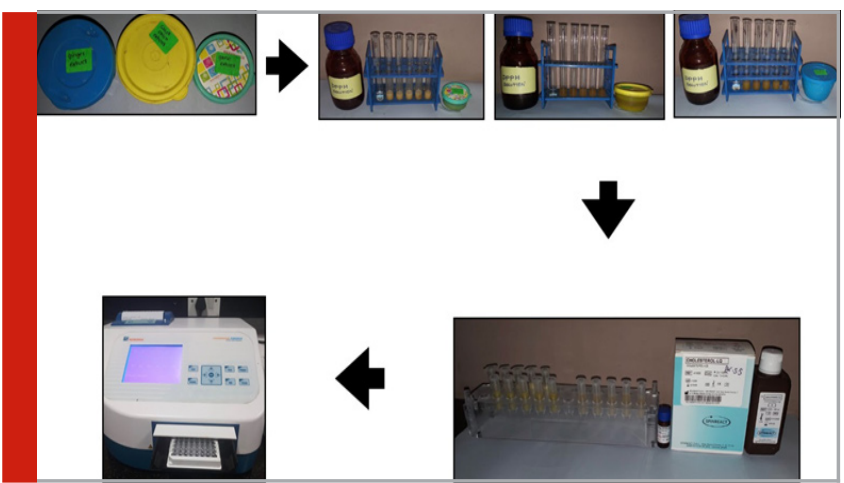




\section{MATERIAL AND METHODS}

Pure extracts of ginger, garlic and small onions were prepared followed by its evaluation of antioxidant potential by using DPPH Free radical scavenging activity, Nitric oxide radical scavenging activity and In vitro anti cholesterol activity. The results thus obtained were statistically compared with each other and also with the combination of the three extracts.

DPPH Free Radical Scavenging Activity: Scavenging of 2,2-Diphenyl-1-picrylhydrazyl (DPPH) radical was assessed by the method of Hatano et al, (1989). DPPH solution $(1.0 \mathrm{ml})$ was added to $1.0 \mathrm{ml}$ of extract at different concentrations $(0.1$ to $0.5 \mathrm{mg} / \mathrm{ml})$. The mixture was kept at room temperature for 50 minutes and the activity was measured at $517 \mathrm{~nm}$. Ascorbic acid at the same concentrations was used as standard. The capability to scavenge the DPPH radical was calculated and expressed in percentage (\%) using following formula:

\section{DPPH radical scavenging $(\%)=$ control OD - sample OD $\times 100$ \\ control OD}

Nitric oxide radical scavenging activity: Scavenging of nitric oxide radical was assayed by the method of Garratt et al. (1964). In the total volume of $3 \mathrm{ml}$ reaction mixture, $2 \mathrm{ml}$ of sodium nitroprusside, $500 \mu \mathrm{l}$ of

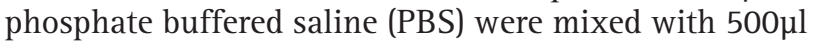
of different concentrations ( 0.1 to $0.5 \mathrm{mg} / \mathrm{ml}$ ) of extract

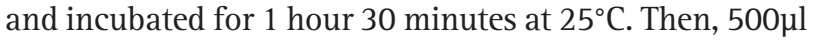
of reaction mixture containing nitrite was mixed with 1 $\mathrm{ml}$ of sulfanilic acid and allowed to stand for 5 minutes for completing diazotization. Then, $1 \mathrm{ml}$ of naphthyl ethylene diamine dihydrochloride was added, mixed and allowed to stand for 30 minutes at $25^{\circ} \mathrm{C}$. Ascorbic acid at the same concentrations was used as standard. The activity was measured at $550 \mathrm{~nm}$ and the results were expressed in percentage (\%) using following formula:

$$
\text { N0 radical scavenging }(\%)=\frac{\text { control OD }- \text { sample OD } \times 100}{-\cdots-\cdots}
$$

In vitro anti-cholesterol activity: The anti-cholesterol assay was carried out as described as per the kit method (Spinreact, S.A.U-Ctra Santa Coloma, Girona, Spain). Cholesterol was dissolved in chloroform at a concentration of $2.5 \mathrm{mg} \mathrm{mL} / \mathrm{ml}$. Ten microliter of the extract was pipetted into a microtiter plate followed by the addition of $2000 \mu \mathrm{L}$ of $\mathrm{R} 1$ reagent and $10 \mu \mathrm{L}$ of cholesterol as sample. Twenty microliter of distilled water and $2000 \mu \mathrm{L}$ of $\mathrm{R} 1$ reagent were used as blank. Negative control consisted of $20 \mu \mathrm{L}$ cholesterol and $2 \mathrm{ml}$ $\mathrm{R} 1$; standard consisted of $20 \mu \mathrm{L}$ simvastatin and 2000 $\mathrm{mL} R 1$ reagent. The contents were incubated between 0-30 min at room temperature and the absorbance was read at $500 \mathrm{~nm}$ in a UV-Vis spectrophotometer against reagent blank. Anti-cholesterol assay of the extract was calculated using the following equation:

$$
\begin{gathered}
\text { Inhibition }(\%)= \\
\text { Negative control-Sample } \times 100 \\
\text { Negative control }
\end{gathered}
$$

Statistical analysis: The data were subjected to statistical analysis using one-way analysis of variance (ANOVA) and Duncan's multiple range test to assess the significance of individual variations between the groups. In Duncan's test, significance was considered at the level of $\mathrm{p}<0.05$.

Figure 1: The bar chart shows the comparison of DPPH free radical scavenging activity of Ginger (Zingiber officinale), Garlic (Allium sativum L.) and small onions (Allium parvum) with the Vitamin C (standard drug). The $\mathrm{X}$ axis represents the concentration in $\mathrm{mg} /$ $\mathrm{ml}$ and the $\mathrm{Y}$ axis represents the \% of inhibition. Dark green colour denotes the standard (vitamin c), Red colour denotes ginger, Blue colour denotes small onions and light green colour denotes garlic. Among the three elements, Ginger (Zingiber officinale) shows higher DPPH free radical scavenging activity when compared to the Garlic (Allium sativum L.) and small onions (Allium parvum).

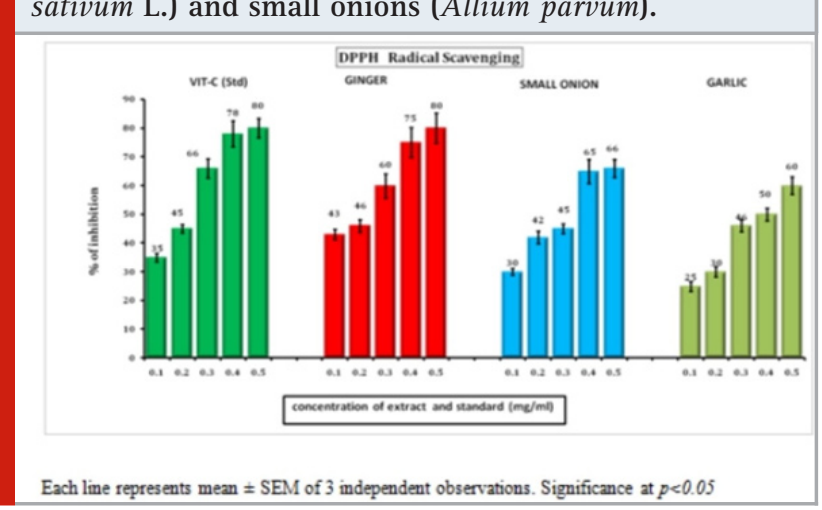

RESULTS AND DISCUSSION

Statistical analysis shows that the allium sativum (garlic) has the highest anticholesterol activity almost equal to the standard $(85 \%$ inhibition at $0.5 \mathrm{mg} / \mathrm{ml}$ conc) when compared with the Allium parvum (small onions) (65\%inhibition at $0.5 \mathrm{mg} / \mathrm{ml}$ conc) and Zingiber officinale (ginger)(75\%inhibition at $0.5 \mathrm{mg} / \mathrm{ml}$ conc ( from Figure 3). In the DPPH free radical scavenging activity analysis, we can able to find that Zingiber officinale (ginger) has the highest antioxidant activity(80\%inhibition at $0.5 \mathrm{mg} /$ $\mathrm{ml}$ conc) when compared to the other two, followed by Allium parvum (small onions)(66\%inhibition at $0.5 \mathrm{mg} /$ $\mathrm{ml}$ conc) and the least antioxidant activity was shown by the Allium sativum (garlic)(60\%inhibition at $0.5 \mathrm{mg} / \mathrm{ml}$ conc) (From Figure 1). Similar antioxidant activities were obtained in cases of nitric oxide free radical scavenging activity also. (From Figure 2).

Interpretation of the polyherbal formulation of the extracts (ginger,garlic and small onions in the ratio 1:2:1) reveals that the combination extracts show an equal (or 
even slightly greater in higher concentrations) when compared to the standards. (From Figure 4,5\&6).with antioxidant activity of (90\%inhibition at $0.5 \mathrm{mg} / \mathrm{ml}$ conc) and anticholesterol activity of (95\%inhibition at $0.5 \mathrm{mg} /$ $\mathrm{ml}$ conc), which are much greater than the individual extracts and also the standard Vit C and Agarbose. So to conclude the results, we can say that allium sativum (garlic) has the individual anti cholesterol and antioxidant activity a little more when compared to other two, while zingiber officinale (ginger) shows more antioxidant activity when compared to the other two extracts. Allium parvum (Small onion), has almost equal anti cholesterol and antioxidant activity. The combination of the extracts in the ratio 1:2:1 (garlic:ginger:small onion) shows almost equal anti cholesterol and antioxidant potential when compared to the standard.

Figure 2: The bar chart shows the comparison of Nitric oxide free radical scavenging activity of Ginger (Zingiber officinale), Garlic (Allium sativum L.) and small onions (Allium parvum) with the Vitamin C (standard drug). The $\mathrm{X}$ axis represents the concentration in $\mathrm{mg} / \mathrm{ml}$ and the $\mathrm{Y}$ axis represents the $\%$ of inhibition. Dark green colour denotes the standard (vitamin c), Red colour denotes ginger, Blue colour denotes small onions and light green colour denotes garlic. Among the three elements, Ginger (Zingiber officinale) shows higher Nitric oxide free radical scavenging activity when compared to the Garlic (Allium sativum L.) and small onions (Allium parvum).

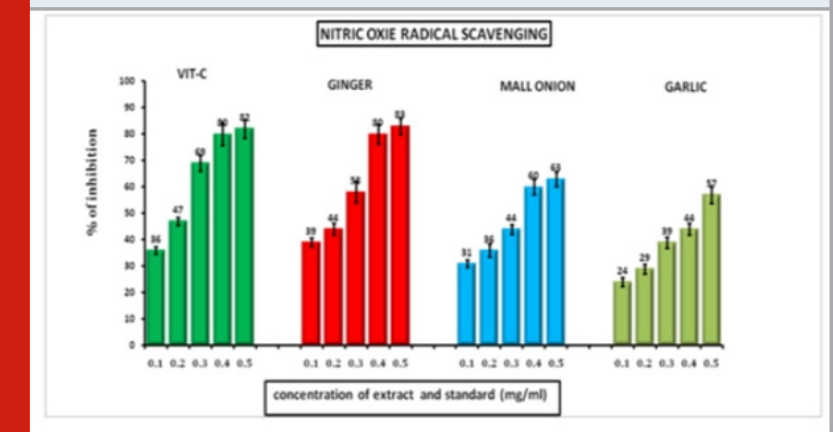

Each line. Represents. Mean SEM of 3 independent observations, Significance. at $p .0 .05$.

Also, Lu et al., have found an important radical scavenging activity of Allium species(Lu et al., 2011). Our results were also comparable to those found by singh et al in 2017 who found that both aqueous and methanolic extracts of Allium sativum have an important anti cholesterol Activity, with better efficiency than the standard used. (Singh and Kumar, 2017) the $\beta$-carotene bleaching test, the antioxidant effect of Zingiber officinale and Allium parvum observed by chung et al in 2019 is perfectly consistent with ours; in fact, He also found that ginger extracts possessed antioxidant activities via the $\beta$-carotene/linoleate system. Likewise, the study noticed a significant hypolipidemic effect substantiated by a notable decline in plasma lipid profile with respect to Allium parvum and also who all have reached a common conclusion which is that the Allium species have a very noticeable hypolipidemic effect (Chung et al., 2019).
Limitations of the study is the fact that the study was conducted in vitro, so it cannot be assumed that the results of anti cholesterol and antioxidant activity could be translated into clinical effectiveness.

Figure 3: The bar chart shows the comparison of Anticholesterol activity of Ginger (Zingiber officinale), Garlic (Allium sativum) and small onions (Allium parvum) with the Agarbose (standard drug). The $\mathrm{X}$ axis represents the concentration in $\mathrm{mg} / \mathrm{ml}$ and the $\mathrm{Y}$ axis represents the $\%$ of inhibition. Dark green colour denotes the standard (Agarbose), Red colour denotes ginger, Blue colour denotes small onions and light green colour denotes garlic. Garlic (Allium sativum) shows higher Anticholesterol activity when compared to the Ginger (Zingiber officinale) and small onions (Allium parvum).

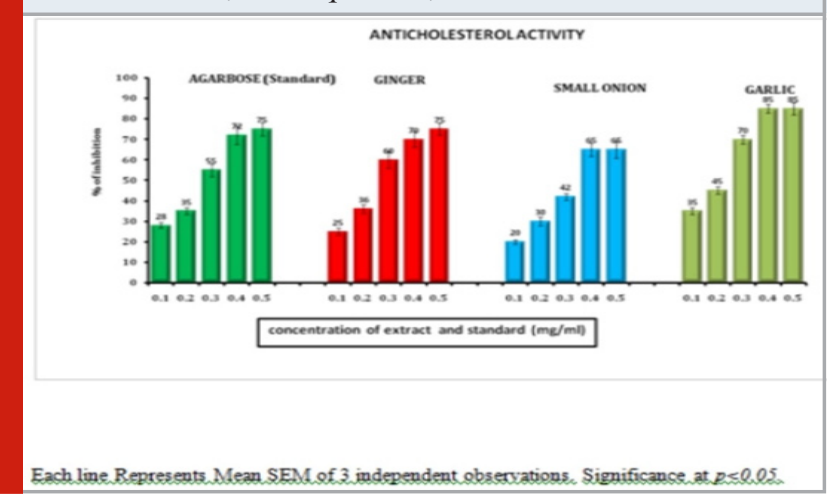

Figure 4: The bar chart shows the comparison of DPPH free radical scavenging activity of Ginger (Zingiber officinale), Garlic (Allium sativum) and small onions (Allium parvum) reinforced together as a polyherbal formulation with the Vitamin $\mathrm{C}($ standard drug). The $\mathrm{X}$ axis represents the concentration in $\mathrm{mg} / \mathrm{ml}$ and the $\mathrm{Y}$ axis represents the $\%$ of inhibition. Green colour denotes ginger, small onions and garlic extracts together as a poly herbal formulation and yellow colour denotes the standard (Vitamin C). It is observed for the graph that the polyherbal formulation shows almost equal DPPH free radical scavenging activity when compared to the standard.

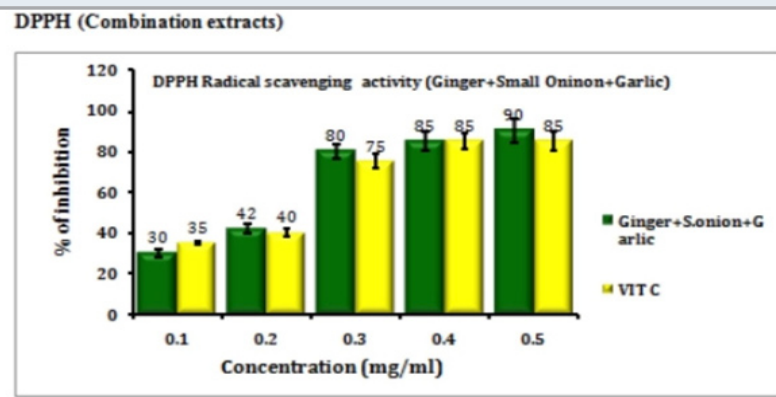

Each lime represents mean $=\mathrm{SEM}$ of 3 independent observations. Significance at $p<0.05$

The significance of our research however, when compared to other articles is that we have also found out the anti cholesterol and antioxidant activity of the polyherbal formulation i.e, a mixture of the ginger, garlic and small onion extracts in the ratio $1: 2: 1$. This throws a new 
limelight on the beneficial effects of not only a single plant extract, but a mixture of plant extracts. The above results show that including ginger, garlic and small onion extracts in regular diets can boost the health to a great extent and prevent early occurring disorders prevalent in the present generation which goes hand in hand with the old saying, "prevention is better than cure".

Figure 5: Figure 5- The bar chart shows the comparison of Nitric oxide free radical scavenging activity of Ginger (Zingiber officinale), Garlic (Allium sativum) and small onions (allium parvum) reinforced together as a polyherbal formulation with the Vitamin C(standard drug). The $\mathrm{X}$ axis represents the concentration in $\mathrm{mg} / \mathrm{ml}$ and the $\mathrm{Y}$ axis represents the \% of inhibition. Green colour denotes ginger, small onions and garlic extracts together as a poly herbal formulation and orange colour denotes the standard (Vitamin C). It is observed for the graph that the polyherbal formulation shows almost equal Nitric oxide free radical scavenging activity when compared to the standard.

\section{NO radical (Combination extracts)}

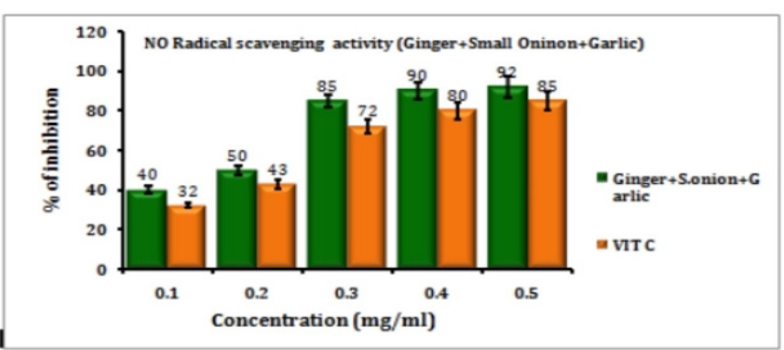

Each line Represents. Mean SEM of 3 independent obseryations, Significance at p. Q Q . 5.

Figure 6: The bar chart shows the comparison of Anticholesterol activity of Ginger (Zingiber officinale), Garlic (Allium sativum) and small onions (Allium parvum) reinforced together as a polyherbal formulation with the Agarbose (standard drug). The $\mathrm{X}$ axis represents the concentration in $\mathrm{mg} / \mathrm{ml}$ and the $\mathrm{Y}$ axis represents the $\%$ of inhibition. Green colour denotes ginger, small onions and garlic extracts together as a poly herbal formulation and Red colour denotes the standard (Agarbose). It is observed for the graph that the polyherbal formulation shows slightly higher Anticholesterol activity when compared to the standard.

ANTI CHOLESTEROL (COMBINATED EXTRACTS)

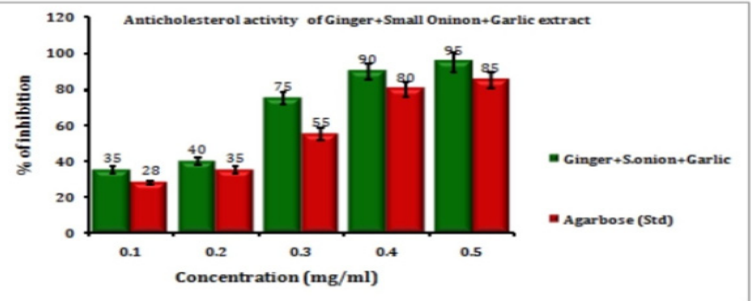

Each line Represents. Mean SEM of 3 independent observations. Significance. at $p \leq 0.05$.

\section{CONCLUSION}

This study has revealed that the extracts of garlic, ginger and small single or as a mixture, possess high polyphenolic content and high antioxidant, anti cholesterol activities in different systems providing support for their acclaimed health benefits. Therefore, further study of their effectiveness in animal models of disease and oxidative stress would be undertaken to provide a useful basis for nutritional advice. Currently there is considerable interest in natural antioxidants and anti cholesterol agents to replace the synthetic ones. "Take healthy food as medicine, before you start taking medicine as food".

\section{ACKNOWLEDGEMENTS}

The authors would like to acknowledge the help and support rendered by the Department of Biochemistry and the Management of Saveetha Dental College and Hospitals for their constant assistance with the research.

Conflict of Interests: None declared.

\section{REFERENCES}

Afzal, M. et al. (2001) 'Ginger: An Ethnomedical, Chemical and Pharmacological Review’, Drug Metabolism and Drug Interactions. doi: 10.1515/dmdi.2001.18.3-4.159. Chung, H. Y. et al. (2019) 'Role of Garlic and Ginger in Anti-oxidative and Anti-inflammatory Effects in Aging', SDRP Journal of Food Science \&t Technology, pp. 788-795. doi: 10.25177/jfst.4.5.mr.533.

Co, E. and Eleazu, C. O. (2013) 'Chemical Composition and Free Radical Scavenging Activities of 10 Elite Accessions of Ginger (Zingiber officinale Roscoe)', Journal of Clinical Toxicology. doi: 10.4172/21610495.1000155.

Dugasani, S. et al. (2010) 'Comparative antioxidant and anti-inflammatory effects of [6]-gingerol, [8]gingerol, [10]-gingerol and [6]-shogaol', Journal of Ethnopharmacology, pp. 515-520. doi: 10.1016/j. jep.2009.10.004.

Ferguson, I. K. and Everett, T. H. (1982) 'The New York Botanical Garden Illustrated Encyclopedia of Horticulture Vol. 3', Kew Bulletin, p. 862. doi: $10.2307 / 4117924$.

Gaafar, M. R. (2012) 'Efficacy of Allium sativum (garlic) against experimental cryptosporidiosis', Alexandria Journal of Medicine, pp. 59-66. doi: 10.1016/j. ajme.2011.12.003.

Gull, I. et al. (2012) 'Inhibitory effect of Allium sativum and Zingiber officinale extracts on clinically important drug resistant pathogenic bacteria', Annals of Clinical Microbiology and Antimicrobials, p. 8. doi: 10.1186/1476-0711-11-8. 
Lanzotti, V. et al. (2012) 'Antifungal saponins from bulbs of garlic, Allium sativum L. var. Voghiera', Phytochemistry, pp. 126-134. doi: 10.1016/j.phytochem.2012.03.009. Leuschner, R. G. K. and Ielsch, V. (2003) 'Antimicrobial effects of garlic, clove and red hot chilli onListeria monocytogenesin broth model systems and soft cheese', International Journal of Food Sciences and Nutrition, pp. 127-133. doi: 10.1080/0963748031000084070.

Li, M. et al. (2017) 'Comparison of Immunomodulatory Effects of Fresh Garlic and Black Garlic Polysaccharides on RAW 264.7 Macrophages', Journal of Food Science, pp. 765-771. doi: 10.1111/1750-3841.13589.

Lu, X. et al. (2011) 'Determination of Total Phenolic Content and Antioxidant Activity of Garlic (Allium sativum) and Elephant Garlic (Allium ampeloprasum) by Attenuated Total Reflectance-Fourier Transformed Infrared Spectroscopy', Journal of Agricultural and Food Chemistry, pp. 5215-5221. doi: 10.1021/jf201254f.

Micová, M. et al. (2018) ‘Content of bioactive compounds and antioxidant activity in garlic (Allium sativum L.)', Acta agriculturae Slovenica, p. 581. doi: 10.14720/ aas.2018.111.3.07.

Ogodo, A. C. and Ekeleme, U. G. (2013) 'In-vitro antibacterial activity of garlic cloves and ginger rhizomes on food-borne pathogens', International Journal of Basic and Applied Sciences. doi: 10.14419/ ijbas.v2i4.1347.

Perry, C. C. et al. (2009) 'Atomic force microscopy study of the antimicrobial activity of aqueous garlicversusampicillin againstEscherichia coliandStaphylococcus aureus', Journal of the Science of Food and Agriculture, pp. 958-964. doi: 10.1002/ jsfa.3538.

Piscitelli, S. C. et al. (2002) 'The Effect of Garlic Supplements on the Pharmacokinetics of Saquinavir', Clinical Infectious Diseases, pp. 234-238. doi: $10.1086 / 324351$.

Prakash, D., Singh, B. N. and Upadhyay, G. (2007)
'Antioxidant and free radical scavenging activities of phenols from onion (Allium cepa)', Food Chemistry, pp. 1389-1393. doi: 10.1016/j.foodchem.2006.06.063.

Prance, G. T., Duke, J. A. and Ayensu, E. S. (1985) 'Medicinal Plants of China', Brittonia, p. 194. doi: $10.2307 / 2806107$.

Qureshi, S. et al. (1989) 'Studies on Herbal Aphrodisiacs Used in Arab System of Medicine', The American Journal of Chinese Medicine, pp. 57-63. doi: 10.1142/ s0192415x89000103.

Santas, J., Almajano, M. P. and Carbó, R. (2010) 'Antimicrobial and antioxidant activity of crude onion (Allium cepa, L.) extracts', International Journal of Food Science \&t Technology, pp. 403-409. doi: 10.1111/j.13652621.2009.02169.x.

Singh, B. N. et al. (2009) 'Polyphenolics from various extracts/fractions of red onion (Allium cepa) peel with potent antioxidant and antimutagenic activities', Food and Chemical Toxicology, pp. 1161-1167. doi: 10.1016/j. fct.2009.02.004.

Singh, V. and Kumar, R. (2017) 'Study of Phytochemical Analysis and Antioxidant Activity of Allium sativum of Bundelkhand Region', International Journal of Life-Sciences Scientific Research, pp. 1451-1458. doi: 10.21276/ijlssr.2017.3.6.4.

Tende, J. A. (2014) 'Effect of Garlic (Allium Sativum) and Ginger (Zingiber Officinale) Extracts on HaematoBiochemical Parameters and Liver Enzyme Activities in Wistar Rats', International Journal of Nutrition and Food Sciences, p. 380. doi: 10.11648/j.ijnfs.20140305.13. Van Damme, E. J. M. et al. (1993) 'Cloning and characterization of the lectin cDNA clones from onion, shallot and leek', Plant Molecular Biology, pp. 365-376. doi: 10.1007/bf00029011.

Ye, C.-L., Dai, D.-H. and Hu, W.-L. (2013) 'Antimicrobial and antioxidant activities of the essential oil from onion (Allium cepa L.)', Food Control, pp. 48-53. doi: 10.1016/j.foodcont.2012.07.033. 